DUKE-CGTP-99-08

hep-th/9910248

October 1999

\title{
Heterotic String Corrections from the Dual Type II String
}

\author{
Paul S. Aspinwall and M. Ronen Plesser \\ Center for Geometry and Theoretical Physics, \\ Box 90318, \\ Duke University, \\ Durham, NC 27708-0318
}

\begin{abstract}
We introduce a method of using the a dual type IIA string to compute $\alpha^{\prime}$-corrections to the moduli space of heterotic string compactifications. In particular we study the hypermultiplet moduli space of a heterotic string on a K3 surface. One application of this machinery shows that type IIB strings compactified on a Calabi-Yau space suffer from worldsheet instantons, spacetime instantons and, in addition, "mixed" instantons which in a sense are both worldsheet and spacetime. As another application we look at the hyperkähler limit of the moduli space in which the K3 surface becomes an ALE space. This is a variant of the "geometric engineering" method used for vector multiplet moduli space and should be applicable to a wide range of examples. In particular we reproduce Sen and Witten's result for the heterotic string on an $A_{1}$ singularity and a trivial bundle and generalize this to a collection of $E_{8}$ point-like instantons on an ALE space.
\end{abstract}




\section{Introduction}

At the present time theories with eight supercharges (such as $N=2$ in four dimensions) represent the "Goldilocks" theories for analyzing moduli spaces of superstring compactifications. With more supersymmetry the moduli space is a symmetric space and in many respects is too rigid to be particularly interesting. With less supersymmetry quantum effects lift some of the classical moduli and computing these is in general not possible with current techniques.

For $N=2$ theories in four dimensions, the moduli space factorizes locally into a product $\mathscr{M}_{V} \times \mathscr{M}_{H}$, where $\mathscr{M}_{V}$ is the special Kähler moduli space coming from scalar fields in vector multiplets and $\mathscr{M}_{H}$ is the hypermultiplet moduli space which is quaternionic Kähler. The space $\mathscr{M}_{V}$ has been studied at great length and can be considered to be fairly well understood. The same is not true for $\mathscr{M}_{H}$.

An important obstacle in the study of $\mathscr{M}_{H}$ can be understood as follows. If we wanted to construct a model producing $N=2$ supersymmetry (complete with gravity) in four dimensions we might try to do it in one of many ways. For example:

1. Compactify the type IIA string on a Calabi-Yau threefold $X$.

2. Compactify the type IIB string on a Calabi-Yau threefold $Y$.

3. Compactify the heterotic string on $S_{H} \times E_{H}$, where $S_{H}$ is a K3 surface and $E_{H}$ is a 2-torus.

In each of these pictures some aspects of the moduli space are exact, in the sense that a computation at string and $\sigma$-model tree level is not corrected by quantum corrections, and some aspects are prone to $\alpha^{\prime}$-corrections, i.e., quantum corrections for the nonlinear $\sigma$-model; or $\lambda$-corrections, i.e., quantum corrections from the effective spacetime quantum field theory (where $\lambda$ represents the string coupling). We list these effects in table 1 .

What is important to note is that the type IIB string on $Y$ gives an exact model for the moduli space $\mathscr{M}_{V}$ whereas none of these pictures gives an exact model for $\mathscr{M}_{H}$. Indeed we do not know any way to get an exact picture of $\mathscr{M}_{H}$. If we could map out $\mathscr{M}_{H}$ then this would be the first time in our study of string theories that we were able to probe truly nonperturbative effects both with respect to $\alpha^{\prime}$ and $\lambda$. It could well be therefore that a better understanding of $\mathscr{M}_{H}$ would lead to a better understanding of the fundamental nature of string theory in general.

What we can see about table 1 is that the compactifications listed above tend to shine light on each other's ignorance about $\mathscr{M}_{H}$. In particular one could try to use the type IIA string to understand the $\alpha^{\prime}$-corrections in the heterotic string compactification.

The general problem of completely analyzing $\mathscr{M}_{H}$ complete with all its quantum corrections is technically very difficult at present although there appears to be nothing lacking in our knowledge in principle. In this paper we will attempt a rather modest goal. For the 


\begin{tabular}{|c||c|c|}
\hline & $\mathscr{M}_{H^{-} \text {-corrections }}$ & $\mathscr{M}_{V^{-}}$-corrections \\
\hline IIA on $X$ & $\lambda$ & $\alpha^{\prime}$ \\
IIB on $Y$ & $\lambda$ and $\alpha^{\prime}$ & Exact \\
Het on $S_{H} \times E_{H}$ & $\alpha^{\prime}$ & $\lambda$ \\
\hline
\end{tabular}

Table 1: Quantum corrections.

latter part we will confine ourselves to the hyperkähler limit of the moduli space where most of the interesting string corrections disappear. We will rederive a result already computed in two different ways by Sen [1] and by Witten [2] and then try to generalize this result. We show that the moduli space of heterotic strings on a blown-up $A_{1}$ singularity, with a trivial bundle near this singularity, is locally described by a hyperkähler limiting manifold of real dimension 4 given by an Atiyah-Hitchin monopole moduli space. Equivalently the moduli space is the same as that of a pure $N=4 \mathrm{SU}(2)$ Yang-Mills theory in three dimensions [3].

We would like to claim that our approach to this problem is useful in many ways. Firstly it is an example of using duality to the type IIA string to obtain nontrivial results about $\alpha^{\prime}-$ corrections to the heterotic string. Secondly, it provides a natural link between the heterotic strings on an ADE singularity and the moduli space of corresponding $N=4$ Yang-Mills theories in three dimensions.

On a third point our method shows that taking the "rigid limit" in which we move from quaternionic Kähler geometry to hyperkähler geometry is mirror to taking the rigid limit of special Kähler geometry in vector multiplet moduli space to obtain Seiberg-Witten theory. Thus the "geometric engineering" methods of [1,5] of analyzing Seiberg-Witten theories can be modified using mirror symmetry to "geometrically engineer" statements about the the hyperkähler moduli space of hypermultiplets in a corresponding limit. This idea of using mirror symmetry together with geometric engineering to obtain a hypermultiplet moduli space plays a role in various contructions that have appeared in the past [6] 9]. In the context of using it to describe vector bundles in the heterotic string it also appears to be closely related to the construction presented in [10].

One should be able to generalize the computation in this paper to many statements about the heterotic string with various bundles on ALE spaces. As an example we "engineer" the problem of one or two free point-like $E_{8}$ instanton coalescing with an $A_{1}$ singularity. This generalizes to give a natural proposal:

Proposition 1 The moduli space of a heterotic string on an ALE space of type $A_{n-1}$ with $k$ point-like $E_{8}$ instantons is same as the moduli space of an $N=4$ gauge theory in three dimensions with gauge group $\mathrm{SU}(n) \times \mathrm{U}(1)^{k}$ and $k$ matter hypermultiplets each in the fundamental representation of $\mathrm{SU}(n)$ and charged with respect to one of the $\mathrm{U}(1)$ 's."

\footnotetext{
${ }^{1}$ To be pedantic this group should probably be written $\mathrm{SU}(n) \times \mathrm{U}(1)^{k} \rtimes \mathrm{H}$, where $\mathrm{H}$ is a discrete group
} 
In principle our method can be used to extend this result to any kind of bundles, including smooth bundles, point-like $\operatorname{Spin}(32) / \mathbb{Z}_{2}$ instantons or fractional point-like instantons as in [11].

It should be emphasized however that this paper should be considered to be the first step in addressing the more interesting question of the full quaternionic Kähler moduli space. In a sense by going to the hyperkähler limit we are avoiding the truly "stringy" nature of the subject and confining ourselves to statements about quantum field theory.

In order to simplify the exposition, this paper will be oriented towards studying a particular example although we will be able to make general statements based on our analysis. It would be nice to study an example where the dimension of $\mathscr{M}_{H}$ is fairly small. This in itself is not particularly easy since simple compactifications of the heterotic string on $S_{H} \times E_{H}$ tend to produce rather enormous $\mathscr{M}_{H}$ 's as seen in [12] for example. Our example will have a moduli space of only 4 quaternionic dimensions.

The general principle of the manipulations in this papers are as follows. First we find a Calabi-Yau threefold $X$ which has a stable degeneration such that the IIA string on $X$ is, at this point, dual to a weakly coupled, large radius limit of the heterotic string configuration we desire. If $Y$ is the mirror of $X$ we will find that, at least in the class of examples we consider, $Y$ is a $\mathrm{K} 3$ fibration with a $\mathbb{P}^{1}$ base. As we move to the stable degeneration of $X$, the base of $Y$ becomes infinitely large. As such the stable degeneration of $X$ is mirror to to the way that Seiberg-Witten theories were "geometrically engineered" in [4 from the type IIA string compactified on $Y$. We will engineer hyperkähler moduli spaces as rigid limits of the quaternionic Kähler manifold $\mathscr{M}_{H}$ rather than the original process of engineering rigid special Kähler moduli spaces as rigid limits of the special Kähler manifold $\mathscr{M}_{V}$.

We introduce the example in section 2 and we compute the stable degeneration required to understand the duality to the heterotic string. We map out the moduli space of this degeneration in section 3 .

In section 4 we discuss what happens if we try to move away from the stable degeneration back into the bulk of the moduli space. The central point of this section is that the K3 surface $S_{H}$ of interest is an elliptic fibration with an infinitely large section and a fibre of zero area. Its volume is finite however and the generic areas of the transcendental 2-spheres are also finite and nonzero.

The picture obtained in section 4 will allow us to make some brief comments about "mixed instantons" in section 5. We argue that there are instanton corrections beyond what one would naïvely consider necessary in superstring compactifications.

In section 6 we then discuss another application of section 4 . Essentially this is analysis of the "first-order" effects as we move back into the bulk of the moduli space. This studies a hyperkähler limit of the general quaternionic Kähler moduli space. In particular this allows us to recover Sen and Witten's result. It is then a simple matter to introduce point-like $E_{8}$

containing as a subgroup the symmetric group on $k$ elements. We will ignore such discrete factors here. 
instantons into the picture and derive proposition 1. We then show how this proposition is linked to Intriligator-Seiberg mirror symmetry in three dimensions.

While we were putting the final touches to this manuscript, [13 appeared which derives proposition [1 by using M-theory arguments.

\section{An Example}

Let us begin with the heterotic/type IIA pair introduced in [12] and later clarified in [14]. We consider $Y$ to be the hypersurface

$$
x_{0}^{2}+x_{1}^{3}+x_{2}^{12}+x_{3}^{24}+x_{4}^{24}=0
$$

in the weighted projective space $\mathbb{P}_{\{12,8,2,1,1\}}^{4}$. The type IIA string on $Y$ is then believed to be dual to an $E_{8} \times E_{8}$ heterotic string compactified on $S_{H} \times E_{H}$ where the gauge bundle is trivial over $E_{H}$ while over $S_{H}$ we have two $E_{8}$ bundles with $c_{2}=10$ and $c_{2}=14$ respectively.

The moduli space $\mathscr{M}_{H}$ is then constructed in the heterotic language from deformations of $S_{H}$ together with its bundle. A K3 surface has 20 quaternionic deformations and an $E_{8}$ bundle has $30 c_{2}-248$ quaternionic deformations. Thus the quaternionic dimension of $\mathscr{M}_{H}$ in this case would be 244 . Alternatively from the type IIA side $\mathscr{M}_{H}$ comes from deformations of complex structure of $Y$, variations of the Ramond-Ramond fields in $H^{3}(Y, \mathrm{U}(1))$ and the dilaton-axion. This gives $h^{2,1}(Y)+1=244$ quaternionic dimensions again.

Clearly it would be a very unwieldy to study an example with this many deformations! In order to cut down to number of hypermultiplets we need to tighten our constraints on the $E_{8}$ bundles and tighten our constraints on the K3 surface $S_{H}$ itself.

Note that this example has $h^{1,1}(Y)=3$. We would be much better off if we took the mirror of $Y$, which we call $X$, and considered the type IIA string on $X$. Now we would have only 4 hypermultiplets. The mirror pair $X$ and $Y$ have been studied extensively [15, 16]. The discussion in [17] is also useful for our purposes.

As well as the usual Greene-Plesser orbifold 18 construction we may also write $X$ in the form of a singular version of $Y$. Let $X$ be the hypersurface in $\mathbb{P}_{\{12,8,2,1,1\}}^{4}$ given by the defining equation

$$
\begin{array}{r}
f_{X}=a_{0} x_{0} x_{1} x_{2} x_{3} x_{4}+a_{1} x_{2}^{6} x_{3}^{6} x_{4}^{6}+a_{2} x_{2}^{5} x_{3}^{7} x_{4}^{7}+ \\
a_{3} x_{2}^{7} x_{3}^{5} x_{4}^{5}+a_{4} x_{2}^{5} x_{3}^{6} x_{4}^{8}+a_{5} x_{2}^{5} x_{3}^{8} x_{4}^{6}+a_{6} x_{1}^{3}+a_{7} x_{0}^{2}=0 .
\end{array}
$$

We implicitly assume that $X$ is blown up to render it smooth. This exhibits an extremal transition from $Y$ to $X$. We now have 3 deformations of complex structure which may be 

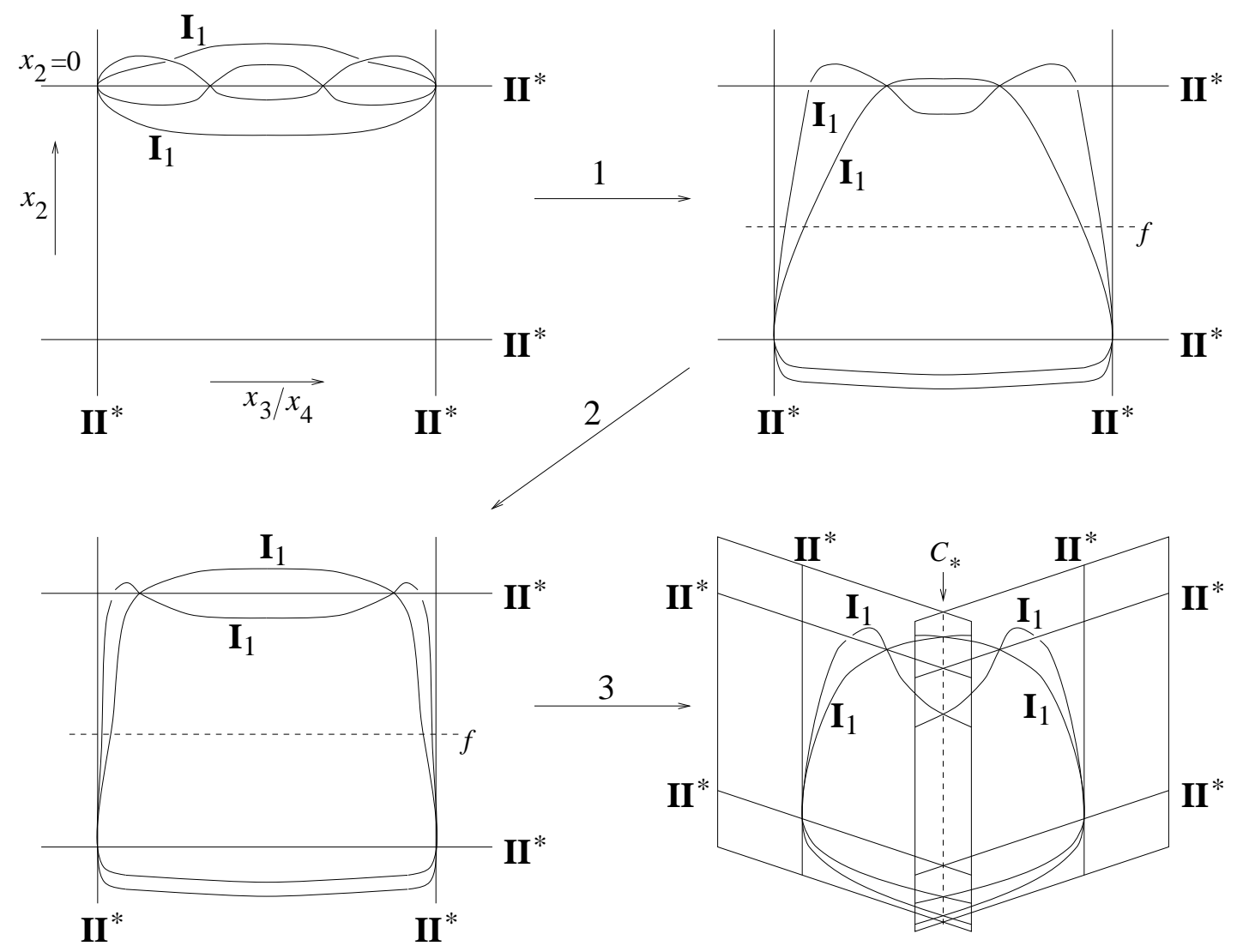

Figure 1: The stable degeneration of $X$.

conveniently represented by

$$
\begin{aligned}
& x=\frac{a_{0}^{6}}{a_{1} a_{6}^{2} a_{7}^{3}} \\
& y=\frac{a_{4} a_{5}}{a_{2}^{2}} \\
& z=\frac{a_{2} a_{3}}{a_{1}^{2}} .
\end{aligned}
$$

Now the stable degeneration method of [19,20] may be used to recover exactly which $E_{8} \times E_{8}$ heterotic string this type IIA compactification represents. In particular we need to find a form of $X$ as a K3 fibration and then find a degeneration which takes each K3 fibre to a union of two rational elliptic surfaces intersecting along an elliptic curve. It is this process which gives a picture of a weakly-coupled heterotic string compactified on a large K3 surface allowing us to make an unambiguous identification of the heterotic string compactification. 
This process is not particularly direct. We depict the manipulations involved in figure 1. The pictures are those typical in F-theory. They show the Calabi-Yau threefold in the form of an elliptic fibration where the plane of the paper represents the base and the lines represent the discriminant locus labelled by the corresponding Kodaira fibre. For more information we refer to [21].

The process is as follows:

1. We begin with $X$. This has four lines of $\mathrm{II}^{*}$ fibres as shown in the upper-left figure. The base is the Hirzebruch surface $\mathbf{F}_{2}$. We wish to change the base to $\mathbf{F}_{0} \cong \mathbb{P}^{1} \times \mathbb{P}^{1}$. This may be done as explained in 14 by blowing up and down curves in the base. In particular we blow up the two top corners of the square in the top-left figure and then blow down the lines corresponding to the left and right sides of the square. This yields the top-right figure. Note that this birational transformation does not change the moduli space of complex structures of $X$.

2. Now we begin to deform the complex structure by taking $y \rightarrow 0$. The interesting parts of the discriminant move towards the vertical sides of the square. If we restrict the elliptic fibration to a horizontal line $f$ we obtain an elliptic K3 surface with two II* fibres and four $\mathrm{I}_{1}$ fibres. As we take $y \rightarrow 0$ the $\mathrm{I}_{1}$ 's move pairwise into the $\mathrm{II}^{*}$ fibres. This is exactly the stable degeneration required as seen in [19].

3. When $y=0$ we obtain very serious singularities along the vertical sides of the square. We may improve matters by blowing up up these sides to produce two new components of the base. Furthermore the original component of the base may be blown down without loss of information. We end up with a base with two components as expected for the stable degeneration picture. The components intersect along a rational curve $C_{*}$. Restricting the elliptic fibration to $C_{*}$ gives a K3 surface $S_{*}$. This surface $S_{*}$ is then isomorphic to the $\mathrm{K} 3$ surface $S_{H}$ on which the $E_{8} \times E_{8}$ heterotic string is compactified.

The standard F-theory rules then tell us that $S_{H}$ is a K3 surface with two $E_{8}$ quotient singularities (as two $\mathrm{II}^{*}$ fibres pass through $C_{*}$ ). Furthermore, the rules in [20] tell us that all 24 instantons are point-like and 10 of them sit at one of the singularities and 14 sit at the other singularity.

This shows explicitly why we have only 4 hypermultiplets. 16 of the 20 deformations of the K3 surface have been fixed by demanding two $E_{8}$ singularities. All deformations of the bundle have been fixed by stating that the instantons are point-like and that their locations are fixed. We assume that $X$ has been blown up, i.e., the corresponding vector or tensor moduli have been switched on to move us into the "Coulomb phase" thus freezing all other hypermultiplet moduli.

It is perhaps worth noting that this stable degeneration and resulting heterotic interpretation of $X$ is not standard. In the usual F-theory language of Morrison and Vafa [14, 22] the K3 fibration of $X$ is given by restricting the elliptic fibration to vertical lines in figure 1. We 
have restricted the elliptic fibration to horizontal lines $f$. Our approach is therefore related to the conventional picture by heterotic-heterotic duality as discussed in [22 24] where the vertical and horizontal directions are exchanged. This appears to be an essential step if we want the stable degeneration of $X$ to correspond to the mirror of giving the base $\mathbb{P}^{1}$ of $Y$ a very large area.

\section{Mapping out the Completely Classical Limit}

To recap, we are going to study an example where the number of quaternionic moduli for $\mathscr{M}_{H}$ is equal to four. This has three interpretations:

1. A type IIA string (or F-theory) compactified on $X . X$ has three deformations of complex structure as given by (2). The other moduli giving $\mathscr{M}_{H}$ are 8 real R-R moduli and the dilaton-axion.

2. A type IIB string on $Y$, the mirror of $X . Y$ has three deformations of Kähler form given by the size of the original $\mathbb{P}_{\{12,8,2,1,1\}}^{4}$ together with two blow-ups required to smooth $Y$. The other moduli giving $\mathscr{M}_{H}$ are 8 real R-R moduli and the dilaton-axion.

3. A heterotic string on a K3 surface $S_{H}$ (times a 2-torus). $S_{H}$ has two $E_{8}$ singularities leaving only 4 quaternionic deformations of the Ricci-flat metric and $B$-field. The bundle is completely rigid and corresponds to point-like instantons buried in the singular points of $S_{H}$.

Now none of these three pictures gives an exact model for the entire moduli space. The type IIA picture will give an exact picture if we fix the dilaton so that the string coupling is zero. This is the limit we are interested in for this paper.

Let us introduce the notation

$$
\operatorname{Grm}(\Lambda)=\mathrm{O}(\Lambda) \backslash \mathrm{O}(n, m) /(\mathrm{O}(n) \times \mathrm{O}(m))
$$

where $\Lambda$ is a Lorentzian lattice (which may or may not be even and unimodular) which has signature $(n, m)$. Thus the moduli space of type IIA strings on a K3 surface would be written $\operatorname{Grm}\left(\Gamma_{4,20}\right)$, where $\Gamma_{m, n}$ will denote an even unimodular lattice of signature $(n, m)$.

In our case we decompose the lattice $H^{*}\left(S_{H}, \mathbb{Z}\right)$ into $\Gamma_{4,20} \cong \Gamma_{4,4} \oplus \Gamma_{0,8} \oplus \Gamma_{0,8}$ where the latter two lattices represent time-like root lattices of $E_{8}$. In order to fix the two $E_{8}$ singularities and set the corresponding components of the $B$-field equal to zero we should delete these latter parts of the $H^{*}\left(S_{H}, \mathbb{Z}\right)$ lattice as explained in [21] for example. Thus, if there were no $\alpha^{\prime}$-corrections we might expect

$$
\mathscr{M}_{H}=\operatorname{Grm}\left(\Gamma_{4,4}\right)
$$


from the heterotic picture.

To make contact with the type IIA picture we follow the usual F-theory process as proposed in [19,20] (see also [21]). Let us suppose that $S_{H}$ is actually an elliptic fibration with a section. We may do this as follows. The $\Gamma_{4,4}$ in (5) represents $H^{*}\left(S_{H}, \mathbb{Z}\right)$ without the $E_{8} \oplus E_{8}$ part coming from the singularities. Let us split this as $\Gamma_{4,4}=\Gamma_{2,2}^{A} \oplus \Gamma_{2,2}^{T}$. Let us split further $\Gamma_{2,2}^{A}=\operatorname{Pic}\left(S_{H}\right) \oplus H^{0}\left(S_{H}, \mathbb{Z}\right) \oplus H^{4}\left(S_{H}, \mathbb{Z}\right)$, where $\operatorname{Pic}\left(S_{H}\right)=\Gamma_{1,1}$ is the Picard lattice of a generic elliptic K3 surface with a section. Note that $\Gamma_{2,2}^{T}$ represents the "transcendental" 2-cycles of $S_{H}$. These 2-cycles have homology classes which are orthogonal to those of the algebraic curves. Any homology 2-cycle can be split into its algebraic part in $\operatorname{Pic}\left(S_{H}\right)$ and its transcendental part in $\Gamma_{2,2}^{T}$.

We now have a natural embedding

$$
\operatorname{Grm}\left(\Gamma_{4,4}\right) \supset \operatorname{Grm}\left(\Gamma_{2,2}^{A}\right) \times \operatorname{Grm}\left(\Gamma_{2,2}^{T}\right) .
$$

$\operatorname{Grm}\left(\Gamma_{2,2}^{A}\right)$ is a 4 real-dimensional space spanned by the value of the complexified Kähler form $B+i J$ on the fibre and the section of $S_{H}$ respectively. $\operatorname{Grm}\left(\Gamma_{2,2}^{T}\right)$ is a 4 real-dimensional space representing the remaining deformations of complex structures of $S_{H}$ as an elliptic fibration. Note that $\operatorname{Grm}\left(\Gamma_{4,4}\right)$ had 16 real dimensions and so we have lost 8 real dimensions in the process of insisting that $S_{H}$ be an elliptic fibration with a section.

Now start taking limits. First we want to take the type IIA string to be weakly coupled. The dilaton of the type IIA string corresponds to the size of the section of $S_{H}$ [21]. Thus we may take the type IIA string to be weakly coupled by moving in the right direction in $\operatorname{Grm}\left(\Gamma_{2,2}^{A}\right)$ so as to make the area of the section huge.

If we take the other component of the Kähler form to be huge so that the area of the elliptic fibres of $S_{H}$ are also large area then we make every 2-cycle in $S_{H}$ huge (assuming the complex structure is generic). This will remove all $\alpha^{\prime}$ corrections. This process will involve moving in a certain direction in the moduli space of complex structures of the threefold $X$ which results in a stable degeneration of $X$. This stable degeneration is of course the one described in section 2. In this limit we expect the remaining two deformations of complex structure of $X$ to map exactly onto $\operatorname{Grm}\left(\Gamma_{2,2}^{T}\right)$.

Fortunately all the hard work of performing the identification in this example has already been done elsewhere [4, 15, 16]. Let us recall the variables defined by (3). The required stable degeneration is then given by the limit $y \rightarrow 0$. In this limit there is an explicit map between $x$ and $z$ and $\operatorname{Grm}\left(\Gamma_{2,2}^{T}\right)$ given as follows. First note that there is an isomorphism between $\operatorname{Grm}\left(\Gamma_{2,2}^{T}\right)$ and two $j$-lines up to discrete identifications:

$$
\operatorname{Grm}\left(\Gamma_{2,2}^{T}\right) \cong\left(\mathbb{Z}_{2} \times \mathbb{Z}_{2}\right) \backslash\left(\frac{\mathrm{SL}(2, \mathbb{R})}{\mathrm{U}(1)} \times \frac{\mathrm{SL}(2, \mathbb{R})}{\mathrm{U}(1)}\right) .
$$

We refer to 25] for example for details of this isomorphism. Then we may map the variables parametrizing the moduli space of $X$ in (3) to two copies of the $j$-line by letting $j_{1}$ and $j_{2}$ 
be the two solutions of

$$
z j^{2}+(432-x-1728 z) j+x^{2}=0 .
$$

All this looks very similar to previous work done in the vector moduli space such as in Kachru et al. [1]. Indeed we will follow this work further but it is important to remember that we are working in the hypermultiplet moduli space and not the vector multiplet space. In the vector multiplet moduli space case studied in [4] the moduli space given by (7) is the special Kähler moduli space coming from the torus for the heterotic string. In our case this moduli space (7) is the moduli space of complex structures on the K3 surface for the heterotic string. In a sense we are working in the mirror side with respect to [4, 12]. We consider the type IIA string on $X$ whereas [4, 12] consider the type IIB string on the same $X$.

We began with a moduli space of 4 quaternionic dimensions and we have ended up with a 4 real dimensional space in this stable degeneration limit. Let us review the moduli we have fixed or lost by going to this limit. For the type IIA string on $X$ :

1. We have lost the $8 \mathrm{R}-\mathrm{R}$ moduli which have no effect in the zero string coupling limit.

2. In the type IIA picture we have fixed the dilaton to zero string coupling and therefore lost the axion which now lives on a circle of zero radius.

3. We have fixed one complex deformation to go the the stable degeneration limit.

Correspondingly in the heterotic string picture:

1. We have fixed the K3 surface $S_{H}$ to be elliptic with a section. This fixes 8 real moduli.

2. We have taken the section to be infinite in area. This kills the effect of the associated $B$-field.

3. We have taken the elliptic fibre to have infinite area. This kills the effect of the associated $B$-field.

In both cases we account for the 12 missing moduli.

\section{Close to the Limit}

Studying a stable degeneration in this case doesn't tell us anything new except for the fact that heterotic/type IIA duality seems to work nicely. It is more interesting if we try to move away from this limit.

In particular what happens if we keep the type IIA string coupling constant zero (or at least very small) but we move away from the stable degeneration? The type IIA picture will 
remain exact since we aren't switching on any $\lambda$-corrections. On the heterotic side however we appear to be allowing for some $\alpha^{\prime}$-corrections to appear.

At first sight you might think that, in terms of the heterotic string, we should study the part of the moduli space where the section of $S_{H}$ is infinite and we give some nonzero value to the size of the fibre. This is not the case and we need to study this process with a little more care.

As Witten argued in [26], any $\alpha^{\prime}$-corrections to the moduli space of the heterotic string should come from world-sheet instantons wrapping themselves around minimal 2-spheres in $S_{H}$. In the stable degeneration of section 2 we have an elliptic K3 surface with infinitely large section and fibre. Assuming the complex structure is generic this would imply that every 2-sphere in $S_{H}$ has infinite area and so there are no quantum corrections.

Let us consider more carefully the finite case away from this limit. We will describe the moduli space $\operatorname{Grm}\left(\Gamma_{4,4}\right)$ in terms introduced in [21,27]. Let $\Gamma_{4,4}=\Gamma_{1,1} \oplus \Gamma_{3,3}$ where $\Gamma_{1,1}$ is spanned by a light-like vector $w$ and its dual $w^{*}$. A point in $\operatorname{Grm}\left(\Gamma_{4,4}\right)$ is determined by a space-like 4-plane $\Pi \subset \Gamma_{4,4} \otimes \mathbb{R}$. Let $\Sigma^{\prime}=w^{\perp} \cap \Pi$ be a space-like 3-plane and define the vector $B^{\prime}$ by the fact that $\Pi$ is a spanned by $\Sigma^{\prime}$ and $B^{\prime}$ and $B^{\prime}$ is perpendicular to any vector in $\Sigma^{\prime}$. We normalize $B^{\prime}$ so that $B^{\prime} . w=1$. We then project $\Sigma^{\prime}$ and $B^{\prime}$ into $\Gamma_{3,3} \otimes \mathbb{R}$ to give the space-like 3 -plane $\Sigma$ and the vector $B$. We may write

$$
B^{\prime}=\alpha w+w^{*}+B,
$$

for some real positive $\alpha$.

Thus a point in $\operatorname{Gr}\left(\Gamma_{4,4}\right)$ can be specified by a point in $\operatorname{Gr}\left(\Gamma_{3,3}\right)$, a vector in $\Gamma_{3,3} \otimes \mathbb{R}$ and a real number $\alpha$. Different choices of $w \in \Gamma_{4,4}$ are identified by the $\mathrm{O}\left(\Gamma_{4,4}\right)$ action. For a given $w$, different choices of $w^{*}$ amount to shifts of $B$ by elements of $\Gamma_{3,3}$.

We expect $\Gamma_{3,3}$ to be the integral 2-cohomology of the K3 surface with two $E_{8}$ singularities, $\Sigma$ to represent the Ricci-flat metric on $S_{H}$ in the usual way, $B$ to represent the $B$-field and the volume of the $\mathrm{K} 3$ surface to be given by $2 \alpha+B^{2}$ [21].

The 3-plane $\Sigma$ represents the directions in $H^{2}$ spanned by the real and imaginary part of the holomorphic 2-form of $S_{H}$ and by the Kähler form. Let $\Omega$ represent the two plane spanned by the holomorphic 2 -form and let $J$ be the Kähler form. Thus $\Sigma$ is spanned by $\Omega$ and $J$. Rotating this decomposition of $\Sigma$ into $\Omega$ and $J$ amounts to changing the complex structure while fixing the metric on $S_{H}$.

Let us fix a point in the moduli space $\operatorname{Gr}\left(\Gamma_{4,4}\right)$ and fix a choice of $w$ to give a geometrical interpretation. Let $e$ be any vector in $\Gamma_{3,3}$ such that $e . e=-2$. Let us choose $\Omega$ so that it is orthogonal to $e$. We are always free to do this for a fixed $\Sigma$. Let us suppose that we are in the generic situation where $e$ is then the only such vector orthogonal to $\Omega$. In this case $e$ (or $-e)$ must represent a rational curve in $S_{H}$ (see for example [26] for this argument). Such a rational curve is a minimal 2 -sphere are thus can give instantons. Thus all such -2 vectors (for one choice of sign) give instantons.

\footnotetext{
${ }^{2}$ This volume factor has errors in earlier versions of [21].
} 
To compute the area of such a curve we may simply take the dot product of $e$ with $J$ using the normalization $J . J=2 \alpha+B^{2}$. Thus we may compute the associated area of all of the instantons without too much difficulty in principle from this lattice picture of the moduli space.

If $S_{h}$ is elliptic with a section we may further decompose $\Gamma_{3,3}=\operatorname{Pic} \oplus \Gamma_{2,2}^{T}$. Let Pic $\cong \Gamma_{1,1}$ be spanned by light-like vectors $v$ and $v^{*}$. The complex structure implied by demanding that $S_{H}$ be elliptic with section implies that we choose $\Omega \subset \Gamma_{2,2}^{T} \otimes \mathbb{R}$ and $J \subset$ Pic $\otimes \mathbb{R}$. Let $\tilde{J}=\beta v+v^{*}$, where $\tilde{J}$ is proportional to $J$. This fixes

$$
J=\sqrt{\frac{\left(2 \alpha+B^{2}\right) \beta}{2}} v+\sqrt{\frac{2 \alpha+B^{2}}{2 \beta}} v^{*} .
$$

We are free to declare that the class $v$ represents the elliptic fibre and $v^{*}-v$ represents the section. Thus the area of the fibre is

$$
J . v=\sqrt{\frac{2 \alpha+B^{2}}{2 \beta}},
$$

and the area of the section is

$$
J .\left(v^{*}-v\right)=\sqrt{\frac{\left(2 \alpha+B^{2}\right) \beta}{2}}-\sqrt{\frac{2 \alpha+B^{2}}{2 \beta}} .
$$

So if we take the limit $\alpha \rightarrow \infty$ then both the fibre and the section are infinite for generic $\beta$. Indeed for generic complex structure we see that all 2-spheres in $S_{H}$ are infinite as $J . J$ must be normalized to infinity.

To move back from this limit we may let $\alpha$ be finite. It would be nice if we could still keep the area of the section infinite as this would correspond to keeping the type IIA string weakly coupled. We can see we may do this if we allow $\beta \rightarrow \infty$ while keeping $\alpha$ finite (but large). This is the limit we use in this paper.

Note that in this limit the area of the fibre is now zero! This should not be seen as particularly nasty however as we don't expect the elliptic fibre to generate any world-sheet instantons. What is more interesting is that since $\alpha$ is finite, the normalization of $J$ is finite and thus for generic values of complex structure, the transcendental 2-spheres in $S_{H}$ will have finite and nonzero size. What we have is a rather funny-looking K3 surface which has an enormous 2-sphere as a section, vanishingly small elliptic fibres and transcendental 2 -spheres which can have any size we wish. The volume of the K3 surface is finite.

It is therefore these transcendental 2 -spheres, i.e. the length -2 vectors in $\Gamma_{2,2}^{T}$, which will be of most interest to us. These are the ones which give nontrivial world-sheet instanton effects. 


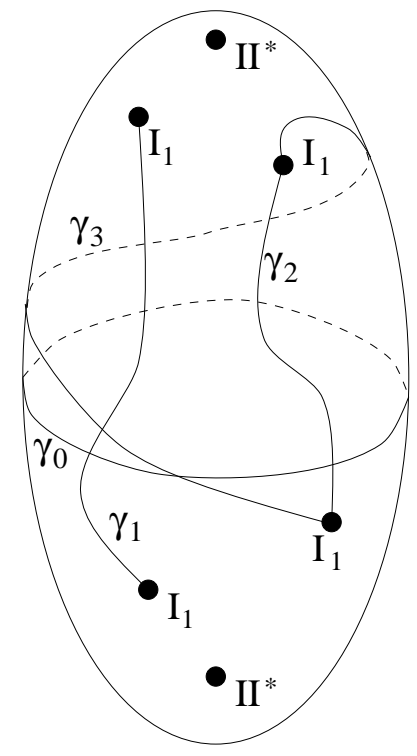

Figure 2: One-chains producing transcendental 2-cycles.

These transcendental 2-spheres may be visualized without too much difficulty in terms described in [28]. Figure 2 depicts the spherical base of the fibration together with the location of the degenerate fibres using Kodaira's notation. Note the two $E_{8}$ singularities given by $\mathrm{II}^{*}$ fibres. Two transcendental 2 -spheres may be made by following the fibration along particular lines joining $\mathrm{I}_{1}$ fibres. The idea is that a circle in the elliptic fibration shrinks to zero size at the $I_{1}$ ends and so this circle sweeps out a 2 -sphere. The paths $\gamma_{1}$ and $\gamma_{2}$ in the figure show such paths. The equatorial path $\gamma_{0}$ in the figure has no monodromy and so we may make two transcendental tori by transporting either of the two circles in the elliptic fibre along this path.

We may also may 2-spheres which are combinations of these classes. For example path $\gamma_{3}$ gives a 2-sphere which is homologically equivalent to the sum of the $\gamma_{2}$ sphere and one of the tori from $\gamma_{0}$.

An important thing to note is that these 2-spheres never see the $\mathrm{II}^{*}$ fibre and as such they miss anything interesting about the the bundle over $S_{H}$. We pushed all the point-like instantons into these II* fibres. Thus the bundle over the 2-spheres we consider for worldsheet instantons is always trivial.

There is an awkward point at this stage which we will evade in this paper but which needs to understood more carefully if we want to understand heterotic worldsheet instantons from type II duality. Consider a transcendental 2-sphere whose class is given by $e \in \Gamma_{2,2}^{T}$. We may then consider an infinity of other 2 -spheres whose class is given by $e+n v$ for any $n \in \mathbb{Z}$. In the limit $\beta \rightarrow \infty$ all of these 2 -spheres will have exactly the same area as the one given by 
e. It appears as though this fact makes any instanton sum divergent. Clearly a more careful treatment of the instanton sum in this limit is required.

\section{Comments on the Type IIB Picture}

In this section we briefly note some peculiarities of the quantum corrections to the type IIB picture of our model. This section is not relevant to the following sections and may be skipped if the reader is only interested in the hyperkähler limits.

As discussed in the introduction, the type IIB string compactified on $Y$ will suffer from both quantum corrections in $\alpha^{\prime}$ and quantum corrections in $\lambda$. It will therefore generically exhibit worldsheet instanton corrections and spacetime instanton corrections. What is perhaps surprising is that our picture in the last section implies that it also exhibits "mixed" instanton corrections depending on $\alpha^{\prime}$ and $\lambda$ simultaneously.

To see this begin with the $\lambda \rightarrow 0$ limit in which the type IIB string has zero coupling and the section $\mathbb{P}^{1}$ of $S_{H}$ is infinitely large. We have worldsheet instantons in the type IIB picture coming from rational curves in $Y$. We also have worldsheet instanton corrections in the heterotic picture coming from transcendental 2-spheres in $S_{H}$. If $Y$ is rescaled to make it larger, the rational curves in $Y$ become large suppressing the worldsheet instanton corrections. In the heterotic picture, the corresponding deformation makes the transcendental 2-spheres in $S_{H}$ larger resulting in a smaller contribution from the worldsheet instantons.

Now let $\lambda$ acquire a small nonzero value for the type IIB string. We know that the type IIB string will now pick up corrections from spacetime instantons. In the heterotic picture the section of $S_{H}$ now becomes finite and we may wrap worldsheet instantons around this $\mathbb{P}^{1}$. Thus the spacetime instantons of the type IIB string appear to map to heterotic worldsheet instantons wrapping the section of $S_{H}$ while the worldsheet instantons of the type IIB string correspond to heterotic worldsheet instantons wrapping the transcendental 2-spheres of $S_{H}$.

As explained by Witten [26] one should get heterotic worldsheet instantons from all the 2spheres in $S_{H}$ (assuming the bundle is suitably trivial). In particular there are infinitely many 2-spheres which are homologically a combination of the section of $S_{H}$ and the transcendental 2 -spheres discussed in the last section. What do heterotic instantons on these "mixed" 2spheres correspond to in the type IIB string? Clearly they give instanton corrections which depend both on $\alpha^{\prime}$ and $\lambda$. As such they are instantons that cannot decide whether they should be considered "worldsheet" or "spacetime" instantons! These mixed instantons must

be taken into account beyond the quantum corrections discussed in [29] for example if one is to obtain an exact answer. 


\section{The Hyperkähler Limit}

One of the main sources of technical difficulties in dealing with the hypermultiplet moduli space $\mathscr{M}_{H}$ is that it is quaternionic Kähler and very little is known about the general structure of quaternionic Kähler spaces. We can be less ambitious in the rest of this paper by only trying to ask about the rigid hyperkähler limit of properties of the moduli space. We should emphasize that up to this point however our method should be perfectly good in principle for determining some properties of the full quaternionic Kähler moduli space.

Taking the hyperkähler limit is completely analogous to asking about the rigid limit of special Kähler geometry. See 30, 31] for example for a discussion of the differences between rigid and nonrigid special Kähler geometry.

Indeed in the very example we are looking at, the rigid limit of the vector multiplet moduli space was studied in [4]. More general analysis of this limit was explored in [5].

In the language of the heterotic string compactified on a K3 surface the hyperkähler limit is understood as follows. We may remove quantum corrections by taking the volume of the K3 surface to infinity for a generic complex structure. Suppose instead we have a K3 surface whose complex structure has been carefully chosen so that one minimal 2-sphere has a small volume. We may try to tune the complex structure so that the 2 -sphere maintains its finite size as we scale the overall volume of the K3 to infinity. What we should be left with is a moduli space for this solitary 2 -sphere sitting in a non-compact space. We may similarly treat a collection of intersecting 2 -spheres. The result is that we replace the moduli space of a compact K3 surface by the moduli space of an ALE space.

Thus we need to zoom in on a part of the moduli space of complex structures where a 2 -sphere is of zero size as we take the stable degeneration limit. This is very easy to do thanks to the work in [4]. In particular we are very close to the analysis of section 6 of [4].

Let us consider a single 2-sphere shrinking down to an $A_{1}$ singularity for the first case. The stable degeneration is $y \rightarrow 0$ as discussed in section 2. We need to tune $x$ and $z$ so as to hit the right part of $\operatorname{Grm}\left(\Gamma_{2,2}^{T}\right)$ to give a vanishing 2 -sphere. We may do this by hitting a generic point on the discriminant of (8)

$$
(4 z-1)\left(864^{2} z-(x-432)^{2}\right)=0 .
$$

If we were talking about vector multiplets as in [4] this would correspond to zooming in on an SU(2) Seiberg-Witten theory.

Let $u$ be the complex variable specifying a generic slice through the $x$ - $z$-plane such that $u=0$ and $y=0$ gives a point on the $\mathrm{SU}(2)$ locus (13). Away from but close to $y=0$ there are two solutions to the discriminant in the $u$-plane. To take the rigid limit we take $y \rightarrow 0$ while rescaling the $u$-plane so as to keep these two solutions at fixed values, say $u= \pm \Lambda^{2}$.

The difference with the hypermultiplet moduli space as opposed to the vector multiplet case is that we need to look at the R-R sector of the type IIA moduli. These R-R fields 
live in the intermediate Jacobian $H^{3}(X, \mathrm{U}(1))$. As such we have a fibration over the above $u$-plane whose fibre is an 8-dimensional torus.

The key point about the rigid limit of special Kähler geometry is that the variation of Hodge structure of a Calabi-Yau threefold is replaced by the variation of a Hodge structure of a Riemann surface [30, 31]. As such, the intermediate Jacobian of the Calabi-Yau threefold is replaced by the Jacobian of the corresponding Riemann surface. This observation was effectively noted in [6, [].

Thus we don't need to do any work to say how the R-R fibres behave as we move about the $u$-plane. The mini-variation of Hodge structure we have zoomed in on by going to the rigid limit ignores the majority of $H^{3}(X, \mathbb{Z})$. We only care about the part which has nontrivial monodromy around the $u$-plane. Thus we only need to consider two of the real R-R fields. These will form an elliptic fibration over the $u$-plane and of course this is exactly the elliptic fibration considered by Seiberg and Witten in their original paper [32].

We arrive at the conclusion that the interesting part of the moduli space describing the acquiring of an $A_{1}$ singularity in the heterotic string consists of a space with 4 real dimensions consisting of the natural elliptic fibration over the Seiberg-Witten plane for an $\mathrm{SU}(2)$ gauge theory (with no flavours).

Given that the moduli space is hyperkähler and thus Ricci-flat, this describes the moduli space precisely except for one thing. We need to specify the area of the elliptic fibre. This is the part of the analysis that is quite difficult in our picture. To do this properly we need to be careful about the rate at which we "zoom in" on the moduli space to obtain the rigid limit while we take the type IIA string to be weakly-coupled. Since analyzing the type IIA string at nonzero coupling is rather difficult we will not attempt such an analysis here. What is more, we already know the answer anyway thanks to Seiberg and Witten [2, 3]. The result is that the elliptic curve should be taken to have infinite area. In this case we recover the Atiyah-Hitchin moduli space of $2 \mathrm{SU}(2)$ monopoles [33]. This is also the same as the moduli space of $N=4 \mathrm{SU}(2)$ Yang-Mills in 3 dimensions.

It should now be immediately clear that we may use this same example to probe the heterotic string near an $A_{2}$ singularity. The point in $\operatorname{Grm}\left(\Gamma_{2,2}^{T}\right)$ which gives such a singularity is given by $j_{1}=j_{2}=0$ and thus by $x=0$ and $z=\frac{1}{4}$. This is exactly the point studied in [4 to recover SU(3) Seiberg-Witten theory. Thus we claim that the corresponding moduli space for hypermultiplets is an abelian fibration over the SU(3) Seiberg-Witten theory where the abelian fibre is given by the Jacobian of the Seiberg-Witten curve for $\mathrm{SU}(3)$ and the volume of this fibre we take to be infinite again. This is the same as the moduli space of $N=4$ $\mathrm{SU}(3)$ Yang-Mills in 3 dimensions by the arguments in [3].

\section{Point-Like Instantons}

We may modify this example to "liberate" one of the point-like $E_{8}$ instantons from one of the singularities in $S_{H}$. In other words we need to take $X$ through an extremal transition 


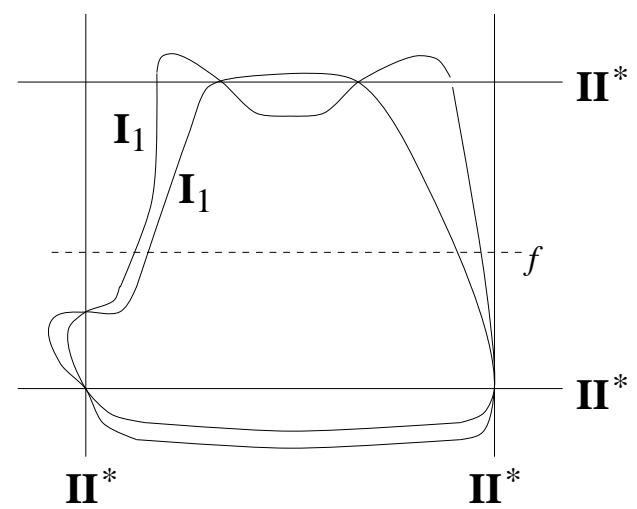

Figure 3: One point-like $E_{8}$ instanton liberated.

to allow one more deformation of complex structure. Let us call this required Calabi-Yau space $X_{1}$. Thus $h^{2,1}\left(X_{1}\right)=h^{2,1}(X)+1$.

We may obtain $X_{1}$ by directly trying to engineer one $E_{8}$ point-like instanton at a generic point on $S_{H}$ while keeping everything the same as the previous situation. From standard Ftheory lore [20,34] we need to replace the upper-right diagram in figure 11 by that in figure 3 . The collision on the left side of the square represents to location of the point-like instanton. This is explained in detail in 35.

Working backwards to the corresponding upper-left picture in figure 1 it is not hard to show that $X_{1}$ is defined by the equation

$$
f_{X_{1}}=f_{X}+a_{8} x_{2}^{6} x_{3}^{7} x_{4}^{5}
$$

where $f_{X}$ is the defining equation of $X$ (written as a singular form of $Y$ ) given by (2).

Thus, the analogue of the geometric engineering story we did in the last section is to consider what theory we would engineer in four dimensions if we compactified the type IIA string on $Y_{1}$, the mirror of $X_{1}$. Our desired moduli space would then correspond to the hyperkähler moduli space given by reducing this four dimensional theory to one in three dimensions.

To understand the geometry of $Y_{1}$ we have depicted a part of the corresponding toric picture in figure 4 . This diagram shows a slice of the toric data representing $Y_{1}$ à la Batyrev [36]. Each vertex represents a divisor in $Y_{1}$ and corresponds to a monomial in the mirror $X_{1}$ [37. We have labelled each vertex accordingly. The vertex labelled by $a_{2}$ represents the divisor given to first approximation by $\mathbb{P}^{1} \times C_{0}$ for some curve $C_{0}$. This is obtained by blowing up the curve $C_{0}$ of $\mathbb{Z}_{2}$-fixed points. The lines $C_{1}$ and $C_{2}$ in the figure represent two $\mathbb{P}^{1}$ 's with normal bundle $\mathcal{O}(-1,-1)$ which replace the generic $\mathbb{P}^{1}$ for a particular point on $C_{0}$. Thus the divisor corresponding to $a_{0}$ is a bundle over $C_{0}$ where every fibre is $\mathbb{P}^{1}$ except for one point where this fibre splits into two $\mathbb{P}^{1}$ 's. 


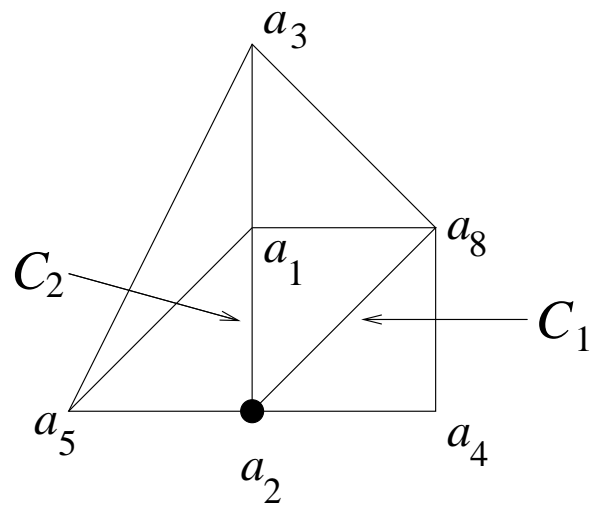

Figure 4: A Toric Picture for $Y_{1}$.

What we have described is precisely the geometric engineering picture of an $\mathrm{SU}(2) \times \mathrm{U}(1)$ gauge theory in four dimensions with a hypermultiplet in the 2 of SU(2) charged with respect to the U(1) [5].

Thus we arrive at the conclusion that the act of letting a free point-like $E_{8}$ instanton "interact" with our $A_{1}$ singularity is tantamount to adding a flavour to the Seiberg-Witten theory and a gauging the associated $\mathrm{U}(1)$ "baryon number" symmetry.

Without much effort one can see that adding the term $a_{9} x_{2}^{6} x_{3}^{5} x_{4}^{7}$ gives us another CalabiYau space $X_{2}$ which corresponds to two point-like instantons floating about the K3 surface $S_{H} . Y_{2}$ then has a ruled surface where one of the fibres becomes a chain of three $\mathbb{P}^{1}$ 's. This is the geometry of the picture of an $\mathrm{SU}(2) \times \mathrm{U}(1)^{2}$ gauge theory in four dimensions with two charged 2's [5].

Although we will not try to prove the general case, the result is fairly self-evident leading to proposition 10 of the introduction. Note in particular that once we have 4 or more pointlike instantons at an $A_{1}$ singularity, we obtain more gauge symmetry and so we have a phase transition leading to more vector multiplets in the four-dimensional theory. This is in perfect correspondence to the fact that Seiberg-Witten theory for $\mathrm{SU}(2) \times \mathrm{U}(1)^{k}$ has a Higgs branch for $k \geq 4$.

It is interesting to note that it was shown in [20] that $k \geq 2 n$ point-like $E_{8}$ instantons coalescing at an $A_{n-1}$ singularity yields a theory with a gauge group in the classical limit given by

$$
\mathscr{G}_{n, k} \cong \mathrm{SU}(2) \oplus \mathrm{SU}(3) \oplus \ldots \oplus \mathrm{SU}(n-1) \oplus \mathrm{SU}(n)^{\oplus(k-2 n+1)} \oplus \mathrm{SU}(n-1) \oplus \ldots \oplus \mathrm{SU}(2),
$$

with bifundamental hypermultiplets charged under each adjacent pair in (15), as well as extra

\footnotetext{
${ }^{3}$ We assume there are no global properties of the compactification leading to non-simply-connected gauge groups.
} 
fundamentals under the two $\mathrm{SU}(2)$ factors and under the first and last $\mathrm{SU}(n)$ 's. As such, once we include quantum corrections we expect the moduli space of the Coulomb branch of our four-dimensional theory to be given by the Seiberg-Witten theory of this group $\mathscr{G}_{n, k}$.

The astute reader will immediately notice that this yields precisely the theory in which in three dimensions is the Intriligator-Seiberg mirror of a theory with gauge symmetry $\mathrm{SU}(n) \times \mathrm{U}(1)^{k}$ 38, 39].

This is explained as follows (see also [8] and particularly [40] for some closely-related comments). Recall the following language for an extremal transition. When in four dimensions the Coulomb phase is part of the moduli space of $\mathscr{M}_{V}$ and is thus special Kähler where the Higgs phase is part of $\mathscr{M}_{H}$ and is thus quaternionic Kähler. If we dimensionally reduce this to three dimensions there is no intrinsic distinction between vector multiplets and hypermultiplets - they both give a quaternionic Kähler moduli space. Even including quantum corrections these multiplets do not mix however and we may still logically label them by their four dimensional origin.

We have argued that the moduli space of $k$ instantons on an $A_{n-1}$ singularity is given by the Coulomb branch of an $\mathrm{SU}(n) \times \mathrm{U}(1)^{k}$ theory in three dimensions. This has a phase transition to a Higgs branch. The results of [20] tell us that this Higgs branch is actually given by the Coulomb branch of a theory with gauge group $\mathscr{G}_{n, k}$ in three dimensions. Thus the Higgs branch of $\mathrm{SU}(n) \times \mathrm{U}(1)^{k}$ is isomorphic to the Coulomb branch of $\mathscr{G}_{n, k}$. This is a statement of Intriligator-Seiberg mirror symmetry. Here we have deduced it from the mirror symmetry of an $X$-type manifold whose stable degeneration represents the heterotic string picture and the mirror $Y$-type which would be used to geometrically engineer the corresponding moduli space.

Note that we could not have used Intriligator-Seiberg mirror symmetry to deduce the examples we discussed for small $k$. Here the theory has no phase transition.

Given the plethora of heterotic string pictures which are understood in F-theory [11, 14, 20] one should be able to extend the analysis of this paper to many more examples.

\section{Acknowledgements}

It is a pleasure to thank S. Katz, D. Morrison, S. Sethi, E. Sharpe and E. Witten for useful discussions. P.S.A. is supported in part by the Alfred P. Sloan Foundation. M.R.P. thanks the Aspen Center for Physics for a stimulating workshop where some of this work was completed.

\section{References}

\footnotetext{
${ }^{4}$ Note that any local analysis along the lines of [40] can often produce extraneous U(1)'s in the gauge group.
} 
[1] A. Sen, Dynamics of Multiple Kaluza-Klein Monopoles in $M$ and String Theory, Adv. Theor. Math. Phys. 1 (1998) 115-126, hep-th/9707042.

[2] E. Witten, Heterotic String Conformal Field Theory and A-D-E Singularities, hepth/9909229.

[3] N. Seiberg and E. Witten, Gauge Dynamics and Compactification to Three Dimensions, hep-th/9607163.

[4] S. Kachru et al., Nonperturbative Results on the Point Particle Limit of N=2 Heterotic String Compactifications, Nucl. Phys. B459 (1996) 537-558, hep-th/9508155.

[5] S. Katz, A. Klemm, and C. Vafa, Geometric Engineering of Quantum Field Theories, Nucl. Phys. B497 (1997) 173-195, hep-th/9609239.

[6] B. R. Greene, D. R. Morrison, and C. Vafa, A Geometric Realization of Confinement, Nucl. Phys. B481 (1996) 513-538, hep-th/9608039.

[7] H. Ooguri and C. Vafa, Summing Up D-instantons, Phys. Rev. Lett. 77 (1996) 32963298, hep-th/9608079.

[8] N. Seiberg and S. Shenker, Hypermultiplet Moduli Space and String Compactification to Three Dimensions, Phys. Lett. B388 (1996) 521-523, hep-th/9608086.

[9] S. Katz, P. Mayr, and C. Vafa, Mirror Symmetry and Exact Solution of $4 D N=2$ Gauge Theories. I, Adv. Theor. Math. Phys. 1 (1998) 53-114, hep-th/9706110.

[10] P. Berglund and P. Mayr, Heterotic String/F-theory Duality from Mirror Symmetry, Adv. Theor. Math. Phys. 2 (1999) 1307-1372, hep-th/9811217.

[11] P. S. Aspinwall and D. R. Morrison, Non-Simply-Connected Gauge Groups and Rational Points on Elliptic Curves, J. High Energy Phys. 07 (1998) 012, hep-th/9805206.

[12] S. Kachru and C. Vafa, Exact Results For N=2 Compactifications of Heterotic Strings, Nucl. Phys. B450 (1995) 69-89, hep-th/9505105.

[13] M. Rozali, Hypermultiplet Moduli Space and Three Dimensional Gauge Theories, hepth/9910238.

[14] D. R. Morrison and C. Vafa, Compactifications of F-Theory on Calabi-Yau Threefolds - II, Nucl. Phys. B476 (1996) 437-469, hep-th/9603161.

[15] A. Klemm, W. Lerche, and P. Mayr, K3-Fibrations and Heterotic-Type II String Duality, Phys. Lett. 357B (1995) 313-322, hep-th/9506112. 
[16] B. H. Lian and S.-T. Yau, Mirror Maps, Modular Relations and Hypergeometric Series I,II, in "S-Duality and Mirror Symmetry", Nucl. Phys. (Proc. Suppl.) B46, pages 248-262, North Holland, 1996, hep-th/9507151, hep-th/9507153.

[17] C. Vafa and E. Witten, Dual String Pairs With $N=1$ and $N=2$ Supersymmetry in Four Dimensions, in "S-Duality and Mirror Symmetry", Nucl. Phys. (Proc. Suppl.) B46, pages 225-247, North Holland, 1996, hep-th/9507050.

[18] B. R. Greene and M. R. Plesser, Duality in Calabi-Yau Moduli Space, Nucl. Phys. B338 (1990) 15-37.

[19] R. Friedman, J. Morgan, and E. Witten, Vector Bundles and F Theory, Commun. Math. Phys. 187 (1997) 679-743, hep-th/9701162.

[20] P. S. Aspinwall and D. R. Morrison, Point-like Instantons on K3 Orbifolds, Nucl. Phys. B503 (1997) 533-564, hep-th/9705104.

[21] P. S. Aspinwall, K3 Surfaces and String Duality, in C. Esthimiou and B. Greene, editors, "Fields, Strings and Duality, TASI 1996", pages 421-540, World Scientific, 1997, hep-th/9611137.

[22] D. R. Morrison and C. Vafa, Compactifications of F-Theory on Calabi-Yau Threefolds - I, Nucl. Phys. B473 (1996) 74-92, hep-th/9602114.

[23] M. J. Duff, R. Minasian, and E. Witten, Evidence for Heterotic/Heterotic Duality, Nucl. Phys. B465 (1996) 413-438, hep-th/9601036.

[24] P. S. Aspinwall and M. Gross, Heterotic-Heterotic String Duality and Multiple K3 Fibrations, Phys. Lett. B382 (1996) 81-88, hep-th/9602118.

[25] A. Giveon, M. Porrati, and E. Rabinovici, Target Space Duality in String Theory, Phys. Rept. 244 (1994) 77-202, hep-th/9401139.

[26] E. Witten, World-Sheet Corrections Via D-Instantons, hep-th/9907041.

[27] P. S. Aspinwall and D. R. Morrison, String Theory on K3 Surfaces, in B. Greene and S.-T. Yau, editors, "Mirror Symmetry II", pages 703-716, International Press, 1996, hep-th/9404151.

[28] P. S. Aspinwall, M-theory Versus F-theory Pictures of the Heterotic String, Adv. Theor. Math. Phys. 1 (1997) 127-147, hep-th/9707014.

[29] K. Becker, M. Becker, and A. Strominger, Five-branes, Membranes and Nonperturbative String Theory, Nucl. Phys. B456 (1995) 130-152, hep-th/9507158. 
[30] D. S. Freed, Special Kähler Manifolds, Commun. Math. Phys. 203 (1999) 31-52, hepth/9712042.

[31] B. Craps, F. Roose, W. Troost, and A. V. Proeyen, What is Special Kähler Geometry?, Nucl. Phys. B503 (1997) 565-613, hep-th/9703082.

[32] N. Seiberg and E. Witten, Electric - Magnetic Duality, Monopole Condensation, and Confinement in N=2 Supersymmetric Yang-Mills Theory, Nucl. Phys. B426 (1994) 19-52, hep-th/9407087.

[33] M. F. Atiyah and N. Hitchin, The Geometry and Dynamics of Magnetic Monopoles, Princeton, 1988.

[34] P. S. Aspinwall, Aspects of the Hypermultiplet Moduli Space in String Duality, J. High Energy Phys. 04 (1998) 019, hep-th/9802194.

[35] P. S. Aspinwall and R. Y. Donagi, The Heterotic String, the Tangent Bundle, and Derived Categories, Adv. Theor. Math. Phys. 2 (1998) 1041-1074, hep-th/9806094.

[36] V. V. Batyrev, Dual Polyhedra and Mirror Symmetry for Calabi-Yau Hypersurfaces in Toric Varieties, J. Alg. Geom. 3 (1994) 493-535.

[37] P. S. Aspinwall, B. R. Greene, and D. R. Morrison, The Monomial-Divisor Mirror Map, Internat. Math. Res. Notices 1993 319-338, alg-geom/9309007.

[38] K. Intriligator and N. Seiberg, Mirror Symmetry in Three Dimensional Gauge Theories, Phys. Lett. B387 (1996) 513-519, hep-th/9607207.

[39] A. Hanany and E. Witten, Type IIB Superstrings, BPS Monopoles, And Three-Dimensional Gauge Dynamics, Nucl. Phys. B492 (1997) 152-190, hep-th/9611230.

[40] K. Hori, H. Ooguri, and C. Vafa, Non-Abelian Conifold Transitions and $N=4$ Dualities in Three Dimensions, Nucl. Phys. B504 (1997) 147-174, hep-th/9705220. 